\title{
Automobile spare-parts forecasting: A comparative study of time series methods
}

\section{C.A. González Vargas* and M. Elizondo Cortés}

\author{
Programa de Posgrado en Ingeniería, Universidad Nacional Autónoma de México, Av. \\ Universidad 3000, Ciudad Universitaria, Coyoacán, \\ Ciudad de México, C.P. 04510, México. \\ *Email: carturo.gvargas@gmail.com \\ Phone: +525526120331
}

\begin{abstract}
In Mexico, the automotive industry is considered to be strategic in the industrial and economic development of the country because it generates production, employment and foreign exchange. Good demand forecasts are needed for better manufacturing management. The time series modelling tools applied to the monthly demand forecasting of automobile spare parts in Mexico are assessed, for the case of a transnational enterprise, considering affordability. The classic methods of moving averages, final value and exponential smoothing, the prestigious autoregressive integrated models of moving averages (ARIMA), the rarely implemented artificial neural networks (ANNs) and the very little explored ARIMA-ANNs hybrid models are compared. A good performance of the models involving ANNs is observed, but they were not as steady as the ARIMA models in the post-sample periods. The mean absolute percentage error (MAPE) was reduced from an original $57 \%$ to $32.65 \%$. The obtained results could help demonstrate the importance of improving industrial forecasting methodologies for better planning.
\end{abstract}

Keywords: Demand Forecasting; supply chain management; forecasting hybrid models; ARIMA-ANN performance comparison; automobile spare parts logistics.

\section{INTRODUCTION}

Nowadays in Mexico, the automotive industry contributes with 3\% to the national Gross Domestic Product (GDP), with $18 \%$ to the manufacturing GDP, and $27 \%$ to the manufacturing exportations [1]. It plays a strategic role in the economy and integrates important national and global production chains. The sector is an important generator of employment and foreign exchange. The automotive industry has operated in the frame of the North American Free Trade Agreement (NAFTA) over the course of 23 years in a good environment, but with the possible renegotiation, the industry as it stands would face new challenges [2]. It is necessary to look for comparative advantages not only in terms of low costs, but also in terms of the research and development capacities required in order to better understand the industry and supply chains trends. The dynamic and uncertain characteristics of the automobile spare-parts demand are critical for national and enterprise economic revenue predictions. Manufacturing and automotive industries are also important in many countries, with their respective share of the business in the spare-parts area. This importance results in strong efforts to achieve a better understanding and their consequent improvements in several areas within the manufacturing and automotive sectors, such as the implementation of quality management systems at different levels [3], surveys on lean manufacturing 
implementation [4], analysis on the influence of the environmental factors in the automotive production processes [5], and even proposals on maintenance management in the sector [6]. Spare parts can be considered as an important business area, with sales revenues ranging from 3.5 to $35 \%$, as found by [7], while studying 7 firms in the engineering industry.

This article is motivated by the necessity of identifying the best method (considering its accuracy and implementing easiness) to face the problem of automobile spare-parts demand forecasting in a transnational enterprise, where a forecasting software is currently being used, giving a sensation of very improvable forecasts to the management, with an average of $57 \%$ of the mean absolute percentage error (MAPE) for their most problematic spare-parts. The study is delimited to its market in Mexico. In the inventories management, for the computation of the economic quantity to order [8], the involved element, which is the most variable and most difficult to compute, is the monthly demand of the product to be manufactured or purchased. As in most enterprises in the automobile spare-parts industry, it is convenient to perform a sales/demand projection and to plan the resources and operative strategies, so as to reduce the logistics cost due to a surplus, or opportunity cost due to the lack of products. In order to satisfy these demands, tools that allow a better management of the inventory in the warehouses of the enterprise that distributes the spare-parts are needed, in an attempt to reduce the costs per inventory, while the demand is satisfied or the management decisions are made [9]. This knowledge will allow flexible responses on behalf of the automobile spare-parts industry to probable changes and the adaptation to new economic conditions. This analysis becomes complex in countries such as Mexico, where few enterprises keep good records of their activities, products and procedures, so that in certain moments within the practice it is mandatory to look for methods which work with the information available, before designing the collection of information, which makes them able to work with better methods.

Improvements in spare parts require that forecasts be searched under different points of view. Some studies have been focused on improving or maintaining service levels [10], while considering inventory metrics as important [11], since special attention should be given to the rapidly increasing number of spare-parts for their correct management [12]. It has been recommended to simplify the existing calculations by implementing simple models when facing big stock keeping units (SKUs) lists [13]. The enterprise under study is considered to require better forecasts to decrease the high logistics costs to which it is exposed. Bacchetti [14] classifies the spare-parts forecast methods in time series, explanatories, hybrids and others, queuing to a selection of the most suitable one, analysing the behaviour of the spare-parts demand under study. For this article, the studied demand is represented by means of time series models, due to the lack of information to model its behaviour explanatorily. Hence, classic time series models are used, due to their simplicity in their elaboration; autoregressive integrated models of moving averages or ARIMA, introduced by Box [15], becoming well-accepted models in the representation of time series; models elaborated through artificial neural networks (ANN) and ARIMA-ANN hybrids, as alternatives to the usual linear modelling in the ARIMA models [16]. With this study, reliable methods for the forecasting of the automobile spare-parts demand are sought to be implemented, in order to obtain estimations and to decide which one can be used in order to achieve the best management of the supply chain and to boost the efficiency of its management. In the second section of this article, a literature review about the spare-parts demand forecasting and a brief theoretical framework about the tools used in this study are presented. In the third part, 
details of the development of the models are exposed, and later, the obtained results are briefed. In the final section, the conclusions of the study are presented.

\section{METHODS AND MATERIALS}

\section{Spare-parts Demand Forecasting}

Notwithstanding the elaboration of forecasts based on the statistics has generated various modelling methodologies, whereas the stochastic nature of various problems found in diverse areas of investigation (industry, public sector, natural phenomena, etc.) makes it convenient to develop better techniques to forecast the specific problem being studied with greater accuracy. In the particular case of spare-parts, the problem has been addressed on the basis of various perspectives, depending on the demand nature and the information available.

When counting on a good data base, with information about the products sold still in use, failure times, lead times, maintenance programs and others, it is possible to estimate the quantity of spare-parts that will be needed over the course of time, by means of an analysis of the installed base [17]. This approach has also been utilised for consumer goods, suggesting that this is a very good way to forecast the demand for spare parts, especially at the end of the life of the product, but also recommends to use simpler methods if the proper information is not currently available [18]. Parallel to this technique that uses no time series, a forecasting method in two steps has been proposed, which separately updates the average of the parts needed per repair and the number of repairs for each sort of component. This method was evaluated in a comparative study for a services provider in the aviation industry [19]. Such investigation takes the advantage of the direct availability of historic information about the repairs of components implying the demand of the specific parts for which the forecast is being performed. Li [20] conducted a study where a decision-making system was developed based on an EFNN (enhanced fuzzy neural network), which is used to forecast the spare-parts demand on the basis of a causal perspective. The aforementioned study takes advantage of the great quantity of information available to the enterprise, since this handles not only the distribution of new automobiles but also the late spare-parts supply itself.

There are useful perspectives for the enterprises which use spare-parts to give internal maintenance to their equipment. Barabadi et al. [21] base their predictions on the statistics models of products' reliability, influenced by factors such as the means of management, maintenance policies, driver's skills, etc., while Lengu et al. [22] propose to discard the supposition of normality in the time series and model the demand of the parts as Bernoulli processes, particularly in the Poisson distributions, taking advantage of the imminent nature of the spare-parts demand. Moon et al. [23] decided to develop a selection model among the forecast methods, in order to predict the performance of each method when forecasting the demand for naval parts in South Korea. Previously developed methods were used, and by means of a logistic regression, it was determined whether one or another method was better for forecasting purposes for the next period. Petropoulos et al. [24], performed a study about the forms of selecting time series forecasting methods, where they suggest that the forecast accuracy is influenced as explained below:

i) For fast-changing data, cyclicality and randomness have the greatest (negative) effect, and the greater the forecast horizons, the greater the decrease in accuracy; 
ii) For intermittent data, the inter-demand interval has a greater (negative) effect than the coefficient of variation;

iii) For every type of data, increasing the length of the series entails small positive effects.

In addition, Sipper and Bulfin [25] propose a methodology to select the demand forecasting methods applied to planning and production management. Even though efficient and complex forecasting methodologies have been developed, there is little evidence of their implementation within the industry [26], and simpler methods, such as the simple moving average or simple exponential smoothing are often used instead [27]. It is also important to identify the nature of the item under study. This can be achieved when classifying them under different criteria, according to our needs and several studies on this matter have been performed, with classifications based on the periodicity of the demand [28], the manufacturing volume [29], or even more complex classifications [30] that should be of help for selecting proper methods and tools for modeling spare-parts behaviour [31]. Based on the literature review up to this point, it was possible to decide that the best tool for modelling the automobile spare-parts demand, given its behaviour and little availability of information, is by means of a time series perspective, so that it has been decided to develop the ARIMA and ANNs models using only the information available in order to obtain forecasts. We can observe a sketched description of the selection process in Figure 1.

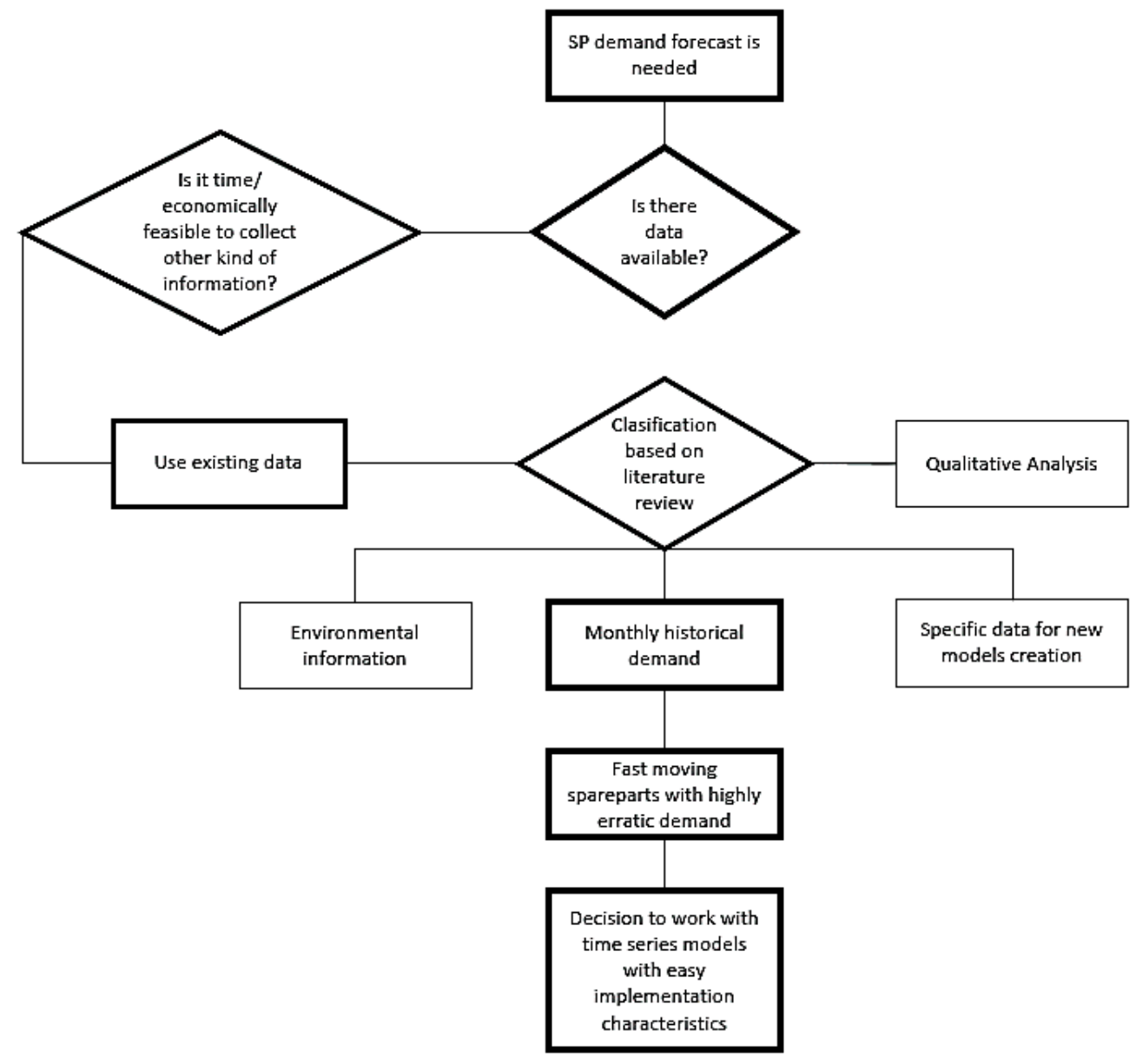

Figure 1. Model selection process, adapted from Sipper and Bulfin's [25]. 


\section{Forecasting with the ARIMA and ANNs Models}

In this study, it is intended to use time series and artificial neural networks as modelling and forecasting tools. There are several studies, which have used these tools in an independent, comparative or combined manner in search of greater accuracy. In recent publications, the comparison of different models for time series forecasting can be observed, involving the ARIMA and ANNs models. In most cases, the ANNs models exceeded the forecasting quality over the ARIMA models [32]; [33]; [34]. Nevertheless, there are also cases in which the ARIMA models worked better [35], and the importance of selecting an appropriate ANNs architecture to solve the corresponding problem is shown [36]. As an alternative, it has been proposed to combine models deploying different methods in order to improve the time series forecasting, commonly obtaining better results than in isolated models [37]; [38]; [39]; [40]; [41]; [42]. Some of these hybrid models have been directly applied to the problem of forecasting spare-parts demand in hybrid industries, combining linear regression and ANNs and working with small sample sizes [43]. Zhang [16] proposes the hybrid models used for this study. He states that the ARIMA and ANNs models have been compared, thus obtaining mixed conclusions in terms performance superiority when forecasting, and therefore proposes a hybrid methodology combining both models in order to take the advantages of one another in linear and nonlinear modelling. The results obtained by Zhang were better in the case of mixed models, than those obtained with each model individually. Several authors have relied on Zhang's models, or have developed models based on the same principle of hybridisation [44]; [44]; [45]; [46].

The ARIMA methodology aims to explain the changes in a time series through the different components forming the ARIMA model $(p, d, q)$ :

- $\operatorname{AR}(p)$, which is the autoregressive component of the model, represents the relationship between the current time series value and its previous values $p$ by means of a linear regression with parameters that weight the influence of the previous values of the times series on the current value. An AR process is only feasible if the time series is stationary and it can be represented as Eq.(1):

$$
Z_{t}=\left(1-\emptyset_{1}-\emptyset_{2}-\cdots-\emptyset_{p}\right) \mu+\emptyset_{1} Z_{t-1}+\cdots+\emptyset_{p} Z_{t-p}+a_{t}
$$

where $Z_{t}$ : is the value of the series for time $t ; \emptyset_{p}$ : are the values of the autoregressive parameters; $\mu$ : is the average, level or expected value of the variable $Z$; $a_{t}$ : is the error or random component for the time $t ; p$ : is the number of delay-periods taking the autoregressive part of the model into account;

$\operatorname{MA}(q)$ is the moving averages component and attempts to explain the current $a_{t}$ error relating it to the $q$ previous errors $a_{t-q}$ in the series. The influence of the previous errors on the last error of the series is weighted by another parameter and this can be represented as Eq. (2):

$$
\widetilde{Z}_{t}=a_{t}-\theta_{1} a_{t-1}-\theta_{2} a_{t-2}-\cdots-\theta_{q} a_{t-q}
$$

where $\tilde{Z}_{t}$ : is the difference between $Z_{t}$ and $\mu$, so the mean and the expected value of $\tilde{Z}_{t}$ are zero; $\theta_{q}$ : are the values of the moving average parameters; $q$ : is the number of the delay-periods taking the moving averages of the model into account.

There are processes that have both an autoregressive and moving averages behaviour; when performing the mathematical development, you get to Eq. (3): 


$$
\tilde{Z}_{t}-\emptyset_{1} \tilde{Z}_{t-1}-\cdots-\emptyset_{p} \tilde{Z}_{t-p}=a_{t}-\theta_{1} a_{t-1}-\cdots-\theta_{q} a_{t-q}
$$

$\mathrm{I}(d)$, which is the difference operation applied $d$ times to the series, aiming to make it stationary, when the original series is not. It calculates the difference between a value of the time series and its previous value, making that difference the new observed value in the series. This action is performed by the application of the operator $\nabla^{d}$, which calculates the difference between each period of the time series a $d$ number of times;

When needed, the stationary process $W_{t}$ can be constructed with Eq.(4):

$$
W_{t}=\nabla^{d} \tilde{Z}_{t}, \text { for every } t .
$$

Finally, the model can be represented as Eq.(5):

$$
W_{t}-\emptyset_{1} W_{t-1}-\cdots-\emptyset_{p} W_{t-p}=\theta_{t}-\theta_{1} a_{t-1}-\cdots-\theta_{q} a_{t-q}
$$

It is understood that a time series is stationary when all its values fit to the same probability distribution, regardless the time. For a deeper study of the methodology, the Box [15] text can be consulted. Meanwhile, artificial neural networks are formed by several layers of nodes which are connected so that in the middle ones, the information which entered via the input nodes is processed and results in useful information being obtained from the output nodes. The designs of either typology depend on the problem to be solved, for instance, to develop a digital filter program in a computer, an algorithm in which all layers are evenly interconnected should be used, meaning that all nodes in a layer are connected to the nodes in another layer [47].

The neural network defines how information processing will be, based on a training process, whereby the weight of each arc connecting two neurons is defined, that is, it ponders the relationship of each input value to each neuron. In the case of the ANN used to forecast, it is normal to try to take advantage of its nonlinear qualities, in contrast to the ARIMA models, which model the time series linearly [35]. Zhang [16] indicates that the most common ANN structure to forecast time series is the single-hidden layer feedforward neural network. Nonetheless, alternatives to selecting a neural network architecture also exist. An architecture designed specifically for obtaining time series forecasting is the NARX architecture (nonlinear autoregressive exogenous network). This network includes delays within its own architecture and has the possibility to include external influences in the model. The equation for the network is Eq.(6):

$$
y(t)=f\left(y(t-1), y(t-2), \ldots, y\left(t-n_{y}\right)\right)
$$

where $f\left(y(t-1), y(t-2), \ldots, y\left(t-n_{y}\right)\right)$ is a nonlinear function of the previous observations during period $t$ and $n_{y}$ is the number of delays taken into account for the model. The output values are recovered and managed as inputs (delays), so a new output value is obtained [48]. In the early stages of this study, better results were obtained using the NARX architecture.

The combination of both methods proposed by Zhang [16] is simple. It states that the forecast can be obtained as Eq.(7):

$$
y_{t}=L_{t}+N_{t}
$$


where $y_{t}$ is the value of the series at time $t, L$ is the linear part of the series and $N$ is the nonlinear part, where the linear part is modelled with ARIMA and the nonlinear with ANNs.

\section{Performance Measures}

As criteria for evaluating the performance of the models, three measures were used. The mean error, which is useful in order to form a clear view on the bias that the forecasts are presenting Eq.(8).

$$
M E=\frac{1}{n} \sum_{t=1}^{n} y_{t}-\hat{y}_{t}
$$

The mean squared error, which penalises large forecast errors as shown in Eq. (9):

$$
M S E=\frac{1}{n} \sum_{t=1}^{n}\left(y_{t}-\hat{y}_{t}\right)^{2}
$$

And the mean absolute percentage error (MAPE), which gives a dimensionless idea of the magnitude of errors as shown in Eq. (10):

$$
M A P E=\frac{1}{n} \sum_{t=1}^{n} \frac{\left|y_{t}-\hat{y}_{t}\right|}{y_{t}}
$$

where $n$ is the total number of periods taken into account.

It is known that the spare-parts demand is frequently intermittent [49], so MAPE is not usually recommended to evaluate their forecasts: for any zero demand, an indeterminate division is obtained [50]. Please note that we found MAPE useful for this study, since no zero-demands were observed and this still provides dimensionless information on the forecasts. An improvement in forecasting can also be seen through better availability rates and shorter delay times [51]. Inventory holdings and inventory costs criteria [52], were not performed due to the lack of information for this particular case, but they might be valuable performance measures depending on the needs of the enterprises.

\section{Calculation}

In order to develop models and draw conclusions from the results, three parts were selected (automobile spare-parts), as they were considered to be especially problematic and representative of the behaviour of other parts regarding their demand in the enterprise being studied. To make the decision about which parts to include in the study, interviews and analyses with the specialist in charge of the enterprise's forecasts, who is considered to be the person most familiar with the problematic, were used. The selected parts were:

- Part A. The behaviour of the demand of this part replicates features of interest for this study, such as a large variability in demand;

- Part B. It is one of the parts whose forecasting errors most affect the enterprise's costs, due to the large number of parts handled and their corresponding physical storage space.

- Part C. This part is characterised by the erratic behaviour of its demand, with significant and unexpected increases and decreases.

For each piece, models with 38 available historic data items at the time of elaboration were developed. This data was supplied by the enterprise under study. Whilst the selection of Box-Jenkins models followed their proposed methodology [15], for all 
other models, the selection was based on their results during the sample period. Moreover, for the analysis of results, the information available was used up to the writing of this article, in order to report an analysis of its implementation in the brief post-sample period at that time. For each part, models were developed as follows:

Classic time series models were developed manually in Microsoft Excel 2010 (Microsoft Corporation), by means of averages and moving averages with up to seven delays and an exponential smoothing applied to the best model obtained among the models previously mentioned. ARIMA models were developed following the methodology suggested by Box and Jenkins [15] and retaken by Guerrero [53], from whom suggestions in the validation/verification techniques of the models were also taken. The software used for the estimation of the parameter was JMP 10.0 (SAS Institute Inc.). The autoregressive and moving average parameters were restricted to those within the stable and invertible regions respectively during the fitting process. ANN models were developed using Matlab R2011a with a NARX feedback architecture with one processing layer for time series modelling. Networks were developed with different numbers of neurons (2-10) and lags (1-7), in order to be later trained and to select the best one for each part. For hybrid models, for each part, the originally selected ARIMA model was taken and a neural network was used (following the same steps for lone ANNs models) in order to try to model the behaviour of the ARIMA model's residuals (the difference between their forecast and real values), as recommended by Zhang [16]. Whilst the development of the classic models is fast and the calculations are performed on a realtime basis, the parameters of the ARIMA model need to be calibrated for each combination of components $(p, d, q)$ considered to represent the system. The ANN need to be developed and trained for each combination of processing neurons and delays, which will take considerably more time to get a good model. For all models, once they have been completely developed, they can be used for forecasting the next periods immediately. The discrepancies between models should also be considered. For classic and ARIMA models, the same parameters should be obtained for a specific time series' sample, but ANN models will depend on the initial training conditions, so different models might be obtained for the same designed structure using the same sample.

\section{Implemented Models}

The resulting models for each part are first presented, in order to later analyse the results in the sample and post-sample periods. The selected model for each part is shown in Table 1 . The sample period spans up to the $38^{\text {th }}$ month and the post-sample period spans up to the $48^{\text {th }}$ month.

Table 1. Architecture for the selected models to each part being studied

\begin{tabular}{|c|c|c|c|c|c|c|c|}
\hline & & & ANN & & Hybrid & & \\
\hline Part & Classic Models & ARIMA & Delays & Neurons & ARIMA & Delays & Neurons \\
\hline A & Average & $\operatorname{ARMA}(3,4)$ & 3 & 7 & $\operatorname{ARMA}(3,4)$ & 2 & 5 \\
\hline B & $\begin{array}{l}\text { Moving averages, } \\
2 \text { delays }\end{array}$ & $\operatorname{ARMA}(5,4)$ & 2 & 8 & $\operatorname{ARMA}(5,4)$ & 2 & 5 \\
\hline $\mathrm{C}$ & $\begin{array}{l}\text { Moving averages, } \\
3 \text { delays }\end{array}$ & $\operatorname{ARMA}(5,4)$ & 3 & 7 & $\operatorname{ARMA}(5,4)$ & 3 & 8 \\
\hline
\end{tabular}




\section{RESULTS AND DISCUSSION}

For the sample period, the combined models were superior to the neural network models; the neural network models were better than the ARIMA models in two out of three cases, and the ARIMA models were also better than the classic models in two out of three cases. In the sample period, more results that are variable can be observed. The demand for part A was steady; therefore the performance indicators were improved. The demand for part B behaved initially steady, but demand peaked at the end, being the hybrid model the one with the most problems to forecast its behaviour since it predicted the demand to become lower instead of higher. The ME, MSE and MAPE for every forecast are shown in Table 2. Part $\mathrm{C}$ had an unexpected behaviour, which could only be represented by the ARIMA model, with a MAPE of $35.91 \%$, against an ANNs of $68.10 \%$, its closest competitor.

Table 2. Performance measures for all parts, for sample and post-sample periods.

\begin{tabular}{llllllll}
\hline \multicolumn{7}{c}{ Period } & \multicolumn{5}{c}{ Post-sample } \\
\hline Part & Model & Sample & \multicolumn{7}{c}{ MAPE } \\
\hline A & & ME & MSE & MAPE & ME & MSE & M. \\
& Classic & 12.64 & $557,480.26$ & $52.72 \%$ & 27.68 & $67,979.62$ & $19.22 \%$ \\
& ARIMA & -28.25 & $379,892.54$ & $38.75 \%$ & -80.42 & $89,392.40$ & $22.34 \%$ \\
& ANN & 60.28 & $337,146.08$ & $35.48 \%$ & -171.64 & $123,931.87$ & $26.09 \%$ \\
& Combined & 22.08 & $310,604.24$ & $29.98 \%$ & -130.5 & $127,926.39$ & $25.99 \%$ \\
B & & & & & & \\
& Classic & -143.36 & $42,053,659.38$ & $32.22 \%$ & $2,941.40$ & $54,948,572.75$ & $21.35 \%$ \\
& ARIMA & 318.86 & $26,204,577.73$ & $25.54 \%$ & $1,164.95$ & $81,944,709.35$ & $39.70 \%$ \\
& ANN & 364.39 & $31,994,816.56$ & $25.81 \%$ & $1,700.39$ & $41,196,172.61$ & $22.85 \%$ \\
& Combined & -91.85 & $17,277,949.33$ & $23.20 \%$ & $1,973.90$ & $139,542,565.57$ & $47.82 \%$ \\
& & & & & & & \\
C & Classic & 26.86 & $65,633.43$ & $29.24 \%$ & -171.98 & $289,281.12$ & $74.86 \%$ \\
& ARIMA & -11.3 & $51,205.87$ & $30.07 \%$ & -36.68 & $103,177.94$ & $35.91 \%$ \\
& ANN & 0.43 & $43,403.78$ & $21.09 \%$ & 195.19 & $477,421.34$ & $68.10 \%$ \\
& Combined & 11.93 & $10,396.58$ & $14.12 \%$ & -321.99 & $331,876.22$ & $71.13 \%$ \\
\hline
\end{tabular}

In Figure 2, the obtained forecasts are shown with each model, for each part and for its respective real demands. Graphically, the behaviour of the real and forecasted demands of each part can be well observed. The erratic behaviour for all parts is clear when looking at their charts. We can also observe the predictions' performances graphically. Figure 3 shows how models have acceptable performances in both periods for parts A and B, whilst the error greatly increased for the post-sample period of part C. From both figures, we can observe that:

- For part A, the demand's variability decreased in the post-sample period, leading to a better performance for all the models if compared with the sample period;

- Part B showed erratic behaviour all along the registered demands, being the most erratic behaviour between months 10 and 14. A similar erratic behaviour was found in the post-sample period, mainly affecting the hybrid model; 
- Part C was the most difficult one to be forecasted, since the most erratic behaviour was found within the transition between the sample and the post-sample period. The only model that remained steady between the two periods was the ARIMA model.
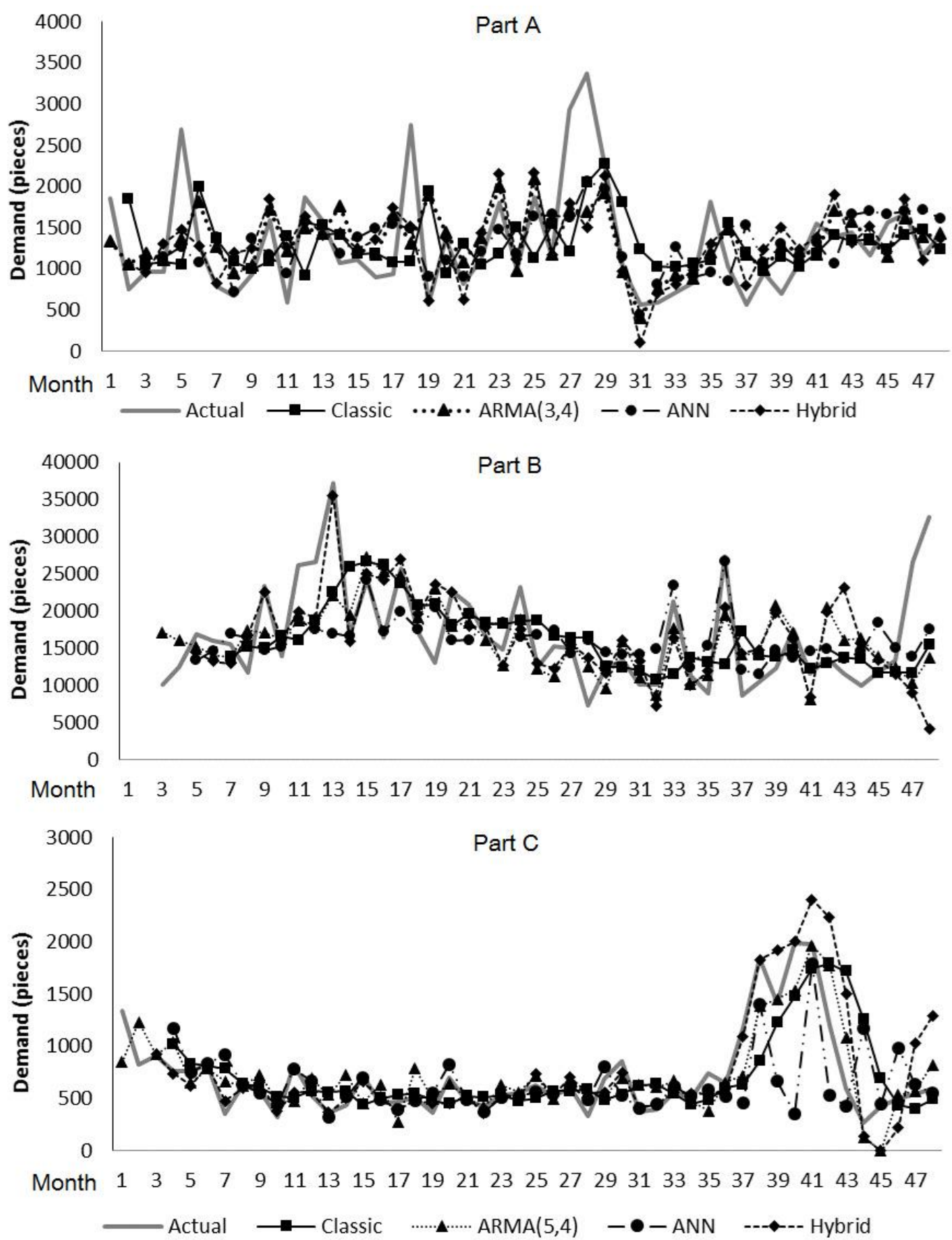

Figure 2. Forecasts for parts A, B and C with all models

Certain advantages and limitations of each modelling method used in the study were observed. For the sample period, the results were very similar to those expected, with the combined models being the superior ones for representing the linear behaviour of the time series with the ARIMA part of the model, and representing the behaviours which had not been captured yet during modelling with the Box and Jenkins 
methodology. In most cases, ANNs performed better than the ARIMA models, and at the same time the ARIMA models were better in their forecast than the classic models. For the post-sample period, mixed results were obtained, which in practice may incur in high costs in the absence of an appropriate interpretation of the behaviour of the models and the real demand. The most interesting contrast between the sample and post-sample periods is the hybrid models' performance. Since the ANN part of Zhang's models seeks to represent the error of the ARIMA model, it is expected to have a better performance than the ARIMA part alone during the sample period, but it will not necessarily have a good performance in the post-sample period. This change can be explained by several different possible factors, such as an overfitting of the model, changes in the demand's behaviour, a small sample, etc. [16]. It is also worth mentioning that the post-sample period up to now involves a very small sample, indicating that little historical data was available, and they were fully used to the development of the models.

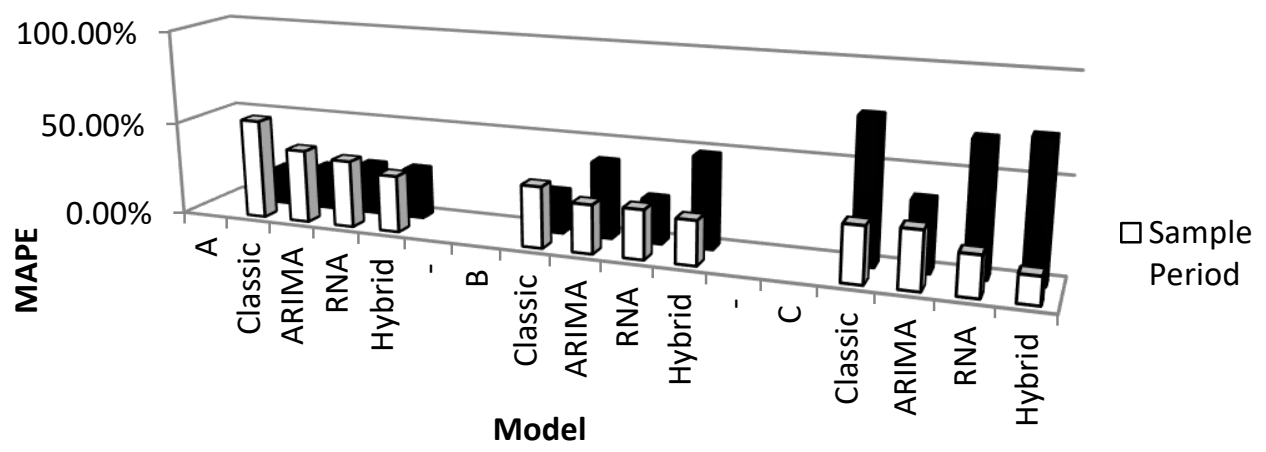

Figure 3. MAPE for each part in the sample and post-sample period.

During the literature review, it was found that for ANN models, much larger historical periods than the ones used in this study are usually handled, but it was decided to work with the information available for this case due to being an alternative to obtaining forecasts. Neural network models are much more sensitive to the sample size because the latter is divided for the training, testing and validation phases; furthermore, due to not being based in a defined mathematical model, they require a greater "expertise" to answering in an appropriate way to the inputs of the model. Owing to these features, neural networks did not have an expected performance for the post-sample period, but stopped capturing the behaviours of the demand, or either overestimated the present behaviours in the sample period, affecting the post-sample forecasts. As previously mentioned, the enterprise reported an average MAPE of $57 \%$ with their current forecasting software. The exercise achieved a significant improvement in the post-sample period, yielding $32.65 \%$ for the best models (ARIMA) and $48.31 \%$ for the ones with the lowest performance (hybrid models). It has been well noted by Granger [54], that some forecasts tend to be very variable too, having too wide prediction intervals. The studied spare parts can be included in this awkward behaviour, but it is better to have an approximation to future demands, so it is worth to maintain efforts for improving our understanding of spare-parts consumption.

As it had been found in previous research [35], in this study diverse results were obtained concerning the convenience of applying either one of the two methods to the case of the automobile spare-parts demand. The results obtained by Hemeimat et al. [31] show that moving averages can provide very competitive forecasts, compared with other techniques, even in the case of exponential smoothing, which has been found not 
advisable to be used for very erratic demands. We obtained similar results and the classic models failed to recognise very erratic demands, as mentioned. Rosienkiewicz et al. [43] obtained better results by using ANNs than with any other time series method, just as we did for the sample periods. Dekker et al. [17] and Kim et al. [18], found good forecasts using the installed base information, but gave no comparison against the time series models. It would be strongly advisable to distinguish the results for the sample and postsample periods in any similar forecasting research study. As future work, expanding the sample period would be recommended for better training of the neural networks, since it has been found that the preference for one method can change depending on the length of the data observation periods [55]; [56].

\section{CONCLUSIONS}

In this paper, some forecasting methods that are affordable for almost any company involved in the automobile spare parts supply chain were assessed. Good alternatives on modelling the spare-parts demand, with no intermittent behaviour, have been assessed. Although no single model outperformed the others for every part and period, their behaviour could be observed. In terms of implementation, classic models are the easiest and fastest to start working with, and provide good results when facing a smooth behaviour in the demand. ANN models were found very promising during the literature review and presented a good fit for the sample period, but started to fail in the post-sample erratic periods. They are the most difficult methods to be implemented, since they require some knowledge of the field in order to have a good selection on the network's architecture and training algorithms. These methods deserve to be further demonstrated, due to the good results obtained with them in other mentioned studies. ARIMA models had the best overall performance and were the only ones to have an acceptable performance during the post-sample erratic period for the piece $\mathrm{C}$. They can be developed in a relatively easy way with some specialised software, but it is important to follow their recommended methodology in order to select the proper model for each time series. During a model selection process, it would be advisable to test models fairly enough prior to implementation. Future research might be focused on improving forecasts through the collection of transcendent information for developing better models. It would also be recommended, for spare-parts traders interested in improving their demand forecasting, to expand their data bases, orientating to the collection of useful information to the development of causal models in order to better evaluate the reasons why each part's demand increases or decreases if the cost/benefit ratio is favourable.

\section{ACKNOWLEDGEMENTS}

The authors would like to thank three anonymous reviewers for their valuable comments for improving this work. The authors are also grateful to Conacyt for funding this research (CVU 559014).

\section{REFERENCES}

[1] Economía-PROMEXICO Sd. La Industria Automotriz Mexicana: Situación actual, Retos y Oportunidades. Ciudad de México: PROMEXICO; 2016.

[2] Legislatura-CEFP CdDL. Boletín: Sector Industrial. La Industria Automotriz en México y el Tratado de Libre Comercio con América del Norte (TLCAN). 2017. 
[3] Civcisa G, Rudnevs J, Grislis A. Quality management systems in small and medium mechanical engineering enterprises in latvia. International Journal of Automotive and Mechanical Engineering. 2014:1928-34.

[4] Rose ANM, Deros BM, Rahman MNA. A study on lean manufacturing implementation in malaysian automotive component industry. International Journal of Automotive and Mechanical Engineering. 2013:1467-76.

[5] Haniff MHM, Ismail AR, Deros BM, Rahman MNA, Kardigama K. The aguchi approach in optimizing environmental factors affecting productivity in the automotive industry. International Journal of Automotive and Mechanical Engineering. 2011:306-17.

[6] Mansor MA, Ohsato A, Sulaiman S. Knowledge management for maintenance activities in the manufacturing sector. International Journal of Automotive and Mechanical Engineering. 2012:612-21.

[7] Wagner SM, Lindemann E. A case study-based analysis of spare parts management in the engineering industry. Production Planning \& Control: The Management of Operations. 2008;19:397-407.

[8] Harris FW. How many parts to make at once. Factory, The Magazine of Management. 1913;10:135-6.

[9] Thomé AMT, Scavadra LF, Pires SRI, Ceryno P, Klingebiel K. A multi-tier study on supply chain flexibility in the automotive industry. International Journal of Production Economics. 2014;158:91-105.

[10] Zhu S, Dekker R, van Jaarsveld W, Renjie RW, Koning AJ. An improved method for forecasting spare parts demand using extreme value theory. European Journal of Operational Research. 2017:169-81.

[11] Ronzoni C, Ferrara A, Grassi A. A stochastic methodology for the optimal management of infrequent demand spare parts in the automotive industry. IFACPapersOnLine. 2015:1405-10.

[12] Morris M. Forecasting challenges of the spare parts industry. Journal of Business forecasting. 2013:22-7.

[13] Tratar LF. Forecasting methos for noisy demand. International Journal of Production Economics. 2015:64-73.

[14] Bacchetti A, Saccani N. Spare parts classification and demand forecasting for stock control: Investigating the gap between research and practice. Omega. 2012;40:722-37.

[15] Box GEP, Jenkins G. Time Series Analysis, Forecasting and Control. San Francisco: Holden-Day; 1970.

[16] Zhang GP. Time series forecasting using a hybrid ARIMA and neural network model. Neurocomputing. 2003;50:159-75.

[17] Dekker R, Pinçe Ç, Zuidwijk R, Jalil MN. On the use of installed base information for spare parts logistics: A review of ideas and industry practice. International Journal of Production Economics. 2013;143:536-45.

[18] Kim TY, Dekker R, Heij C. Spare part demand forecasting for consumer goods using installed base information. Computers \& Industrial Engineering. 2017:20115.

[19] Romeijnders W, Teunter R, Jaarsveld Wv. A two-step method for forecasting spare parts demand using information on component repairs. European Journal of Operational Research. 2012;220:386-93.

[20] Li SG, Kuo X. The inventory management system for automobile spare parts in a central warehouse. Expert Systems with Applications. 2008;34:1144-53. 
[21] Barabadi A, Barabady J, Markeset T. Application of reliability models with covariates in spare part prediction and optimization - A case study. Reliability Engineering and System Safety. 2014;123:1-7.

[22] Lengu D, Syntetos AA, Babai MZ. Spare parts management: Linking distributional assumptions to demand classification. European Journal of Operational Research. 2014;235:624-35.

[23] Moon S, Simpson A, Hicks C. The development of a classification model for predicting the performance of forecasting methods for naval spare parts demand. International Journal of Production Economics. 2013;143:449-54.

[24] Petropoulos F, Makridakis S, Assimakopoulos V, Nikolopoulos K. 'Horses for Courses' in demand forecasting. European Journal of Operational Research. 2014;237:152-63.

[25] Sipper D, Bulfin RL. Planeación y Control de la Producción. México: Mc. Graw Hill; 1998.

[26] Huiskonen J. Maintenance spare parts logistics: Special characteristics and strategic choices. International Journal of Production Economics. 2001;71:12533.

[27] Syntetos AA, Babai Z, Boylan JE, Kolassa S, Nikolopoulos K. Supply chain forecasting: Theory, practice, their gap and the future. European Journal of Operational Research. 2016;252:1-26.

[28] Rego JRd, Mesquita MAd. Demand forecasting and inventory control: A simulation study on automotive spare parts. International Journal of Production Economics. 2015:1-16.

[29] Mohamed NMZN, Khan MK. Decomposition of manufacturing processes: a review. International Journal of Automotive and Mechanical Engineering. 2012:545-60.

[30] Bacchetti A, Plebani F, Saccani N, Syntetos AA. Empirically-driven hierarchical classification of stock keeping units. International Journal of Production Economics. 2013:263-74.

[31] Hemeimat R, Al-Qatawneh L, Arafeh M, Masoud S. Forecasting spare parts demand using statistical analysis. American Journal of Operations Research. 2016:113-20.

[32] Ansuj AP, Camargo ME, Radharamanan R, Petry DG. Sales forecasting using time series and neural networks. Computers \& Industrial Engineering. 1996;31:421-4.

[33] Chattopadhyay S, Chattopadhyay G. Univariate modelling of summer-monsoon rainfall time series: Comparison between ARIMA an ARNN. Comptes Rendus Geoscience. 2010;342:100-7.

[34] Karbasi A, Laskukalayeh Ss, Fahimifard SM. Comparison of NNARX, ANN and ARIMA Techniques to Poultry Retail Price Forecasting. 2009.

[35] Zhang G, Patuwo BE, Hu MY. Forecasting with artificial neural networks: The state of the art. International Journal of Forecasting. 1998;14:35-62.

[36] Ho SL, Xie M, Goh TN. A comparative study of neural network and Box-Jenkins ARIMA modeling in time series prediction. Computers \& Industrial Engineering. 2002;42:371-5.

[37] Díaz-Robles LA, Ortega JC, Fu JS, Reed GD, Chow JC, Watson JG, et al. A hybrid ARIMA and artificial neural networks model to forecast particulate matter in urban areas: The case of Temuco, Chile. Atmospheric Environment. 2008;42:8331-40. 
[38] Goyal P, Chan AT, Jaiswal N. Statistical models for the prediction of respirable suspended particulate matter in urban cities. Atmospheric Environment. 2006;40:2068-77.

[39] Khashei M, Bijari M, Raissi Ardali GA. Hybridization of autoregressive integrated moving average (ARIMA) with probabilistic neural networks (PNNs). Computers \& Industrial Engineering. 2012;63:37-45.

[40] Koutroumanidis T, Ioannou K, Arabatzis G. Predicting fuelwood prices in Greece with the use of ARIMA models, artificial neural networks and a hybrid ARIMAANN model. Energy Policy. 2009;37:3627-34.

[41] Liu H, Tian H-q, Li Y-f. Comparison of two new ARIMA-ANN and ARIMAKalman hybrid methods for wind speed prediction. Applied Energy. 2012;98:41524.

[42] Pektaş AO, Cigizoglu HK. ANN hybrid model versus ARIMA and ARIMAX models of runoff coefficient. Journal of Hydrology. 2013;500:21-36.

[43] Rosienkiewicz M, Chlebus E, Detyna J. A hybrid spares demand forecasting method dedicated to mining industry. Applied Mathematical Modelling. 2017:87107.

[44] Aburto L, Weber R. Improved supply chain management based on hybrid demand forecasts. Applied Soft Computing. 2007;7:136-44.

[45] Pham HT, Tran VT, Yang B-S. A hybrid of nonlinear autoregressive model with exogenous input and autoregressive moving average model for long-term machine state forecasting. Expert Systems with Applications. 2010;37:3310-7.

[46] Zou HF, Xia GP, Yang FT, Wang HY. An investigation and comparison of artificial neural network and time series models for Chinese food grain price forecasting. Neurocomputing. 2007;70:2913-23.

[47] Sotolongo G, Guzmán MV. Aplicaciones de las redes neuronales. El caso de la Bibliometría. Ciencias de la información. 2001;32:27-34.

[48] MathWorks.com. 2014.

[49] Boylan JE, Syntetos AA. Forecasting for Inventory Management of Service Parts. Complex System Maintenance Handbook. 2007; p. 479-506.

[50] Kim S, Kim H. A new metric of absolute percentage error for intermittent demand forecasts. International Journal of Forecasting. 2016;32:669-79.

[51] Leew Sd, Beekman L. Supply chain-oriented performance measurement for automotive spare parts. International Journal of Automotive Technology and Management. 2008;8:56-70.

[52] Ali MM, Boylan JE, Syntetos AA. Forecast errors and inventory performance under forecast information sharing. International Journal of Forecasting. 2012;28:830-41.

[53] Guerrero Guzmán VM. Análisis estadístico y pronóstico de series de tiempo económicas. México: Jit Press; 2009.

[54] Granger CWJ. Can we improve the perceived quality of economic forecasts? Journal of Applied Econometrics. 1996:455-73.

[55] Wang C-C. A comparison study between fuzzy time series model and ARIMA model for forecasting Taiwan export. Expert Systems with Applications. 2011;38:9296-304.

[56] Wong HL, Tu YH, Wang CC. Application of fuzzy time series models for forecasting the amount of Taiwan export. Expert Systems with Applications. 2010;37:1465-70. 